\title{
Relationship Between Matrix Metalloproteinase-9 1562C/T Polymorphism and Liver Diseases: A Meta- Analysis
}

\section{Tianling Yang}

Key Laboratory of AIDS Immunology of National Health and Family Planning Commission

\section{Shitong Cheng}

Department of Laboratory Medicine, the first affiliated hospital of China Medical University

\section{Shumeng Li}

Beijing University of Chinese Medicine

Yangtao Ji ( $\nabla$ ytji@cmu.edu.cn )

Department of Laboratory Medicine, the first affiliated hospital of China Medical University

\section{Research Article}

Keywords: MMP-9, Gene polymorphism, liver disease, meta-analysis

Posted Date: March 10th, 2021

DOI: https://doi.org/10.21203/rs.3.rs-255552/v1

License: (1) (1) This work is licensed under a Creative Commons Attribution 4.0 International License. Read Full License 


\section{Abstract \\ Background}

Liver diseases have an adverse impact on human's body and life, and many factors, such as viruses, genes, etc., are closely related to the occurrence and development of liver diseases. This study tries to explore the relationship between liver diseases and one of the genetic polymorphisms (MMP-9-1562 C/T polymorphism) by meta-analysis, to provide basis for the prevention and treatment of liver diseases and to promote the study of the mechanism of liver diseases by the exploration of etiology.

\section{Methods}

Relevant literatures from PubMed, EMBASE, Web of Science, CNKI, Chinese biomedical literature database and other databases were searched from building the database to Dec. 2020. Meta-analysis method was adopted and Review Manager 5.3 software was used for analysis.

\section{Results}

A total of 7 studies with 1415 cases and 1592 controls were included in this meta-analysis. A significant association between MMP-9-1562 C/T polymorphism and liver diseases in the homozygous and recessive models (TT vs CC: $\mathrm{OR}=1.82,95 \% \mathrm{Cl}=1.28-2.59, P=0.0009$; $\mathrm{TT}$ vs $\mathrm{CT}+\mathrm{CC}$ : $\mathrm{OR}=1.73,95 \% \mathrm{Cl}=1.24-2.41, P=$ 0.001 ). However, no association in heterozygote and dominant models (CT vs CC: $\mathrm{OR}=1.05,95 \% \mathrm{Cl}=0.76-$ $1.45, P=0.78 ; \mathrm{TT}+\mathrm{CT}$ vs CC: $\mathrm{OR}=1.17,95 \% \mathrm{Cl}=0.82-1.67, P=0.38)$.

\section{Conclusions}

Our meta-analysis suggested that the TT genotype of MMP-9-1562 C/T polymorphism might be associated with the risk of nonalcoholic fatty liver disease, hepatocellular carcinoma, and primary liver cancer. If these diseases are present, screening genotype of MMP-9 and aggressive treatment of the primary disease is necessary.

\section{Background}

Liver disease is a common and extremely harmful global disease [1]. In recent years, the incidence and mortality of liver disease have been increasing, which has become a challenging and urgent clinical problem to be solved. Drug toxicity, virus infection, alcohol and other factors will lead to the occurrence of liver disease, and often due to the delay of the treatment time or early neglected, prompting the activation of hepatic stellate cells and liver damage of the structure, and then liver fibrosis, the pathological basis of cirrhosis or cancer occurred. If not timely intervention and treatment, early liver disease may develop irreversible damage namely liver cirrhosis ,even liver cancer.[2]. 
Matrix Metalloproteinases (MMPs), a family of zinc-dependent proteolytic enzymes, which plays a key role in the degradation of ECM and the injury and repair of liver structure, and is also closely related to the progress and repair of liver fibrosis[3]. Matrix Metalloproteinase-9 (MMP-9), also known as gelatinase B [4], is one of the important components of ECM degradation and has a bearing on the dynamic changes of liver fibrosis. The activity of MMP-9 is significantly affected by gene polymorphism. Current studies have identified MMP9-1562 C/T (rs3918242) polymorphism within the promoter of the MMP-9 gene[5], which can vitally affect its function, and thus may be close to various liver diseases.

In order to clarify the relationship between MMP-9-1562C/T (rs3918242) polymorphism and liver diseases, this study conducted a comprehensive meta-analysis (META) on published studies to obtain more reliable conclusions, providing scientific basis for the prevention and treatment of liver diseases and the exploration of etiology to help for the study of the mechanism of liver diseases.

\section{Methods}

\subsection{Literature retrieval}

We searched related articles published in PubMed, EMBASE, Web of Science, CNKI and China Biomedical Literature Database with the retrieval date of Dec. 1, 2020. Retrieval words: "MMP-9, matrix metalloproteinase-9 or gelatinase B", "polymorphism, single nucleotide polymorphism or SNP" and "liver or hepatic".

\subsection{Inclusion and exclusion criteria}

The relationship between MMP-9 polymorphism and liver diseases was analyzed and determine the inclusion criteria: a) case-control study; B) polymorphism of MMP-9 1562; C) liver disease; D) there were odds ratios (ORs) and $95 \%$ confidence intervals (Cls) for polymorphism in the case group and control group. Exclusion criteria: a) case reports, reviews, excerpts, or unpublished data; B) overlapping data; C) research on family members.

\subsection{Data extraction}

Yang Tianling, a student, extracted data independently from all the selected studies, and the differences in the included literature were handled by instructor Ji Yangtao. The following information was extracted: first author, year of publication, race, source of control group, liver disease type, genotyping method, number of case and control group, distribution frequency of MMP-9-1562 polymorphisms CC, CT, TT, and literature quality score.

\subsection{Literature quality evaluation}

For the included case-control studies, the Newcastle-Ottawa Scale (NOS) literature quality evaluation Scale (full score is 9, including object selection, comparability and exposure) was used to conduct methodological quality evaluation. Literature quality was considered to be good if the score was above 5 .

\subsection{Statistical analysis}


Review Manager 5.3 was used for statistical analysis. The association between MMP-9-1562 C/T gene polymorphism and liver disease was examined by odds ratio (OR) and $95 \%$ confidence interval (Cl). Four genetic models were analyzed: homozygous contrast model (TT vs CC), heterozygous contrast model (CT vs $C C)$, dominant model ( $T T+C T$ vs $C C$ ) and recessive model (TT vs $C T+C C)$.

For the included studies, $\mathrm{X}^{2}$ test was used to evaluate the heterogeneity among the studies, and $\mathrm{I}^{2}$ was used to quantitatively analyze the heterogeneity. $I^{2} \leqq 50 \%$ was considered to be small heterogeneity, and fixed effect model was used; $\mathrm{I}^{2}>50 \%$ is expected to exist large heterogeneity among the studies, and random effect model was used. Find the sources of heterogeneity, when $\mathrm{I}^{2}=0$, no heterogeneity. Funnel plot was used for publication bias analysis. $P$ value $<0.05$ was considered statistically significant.

\section{Results}

\subsection{Basic features of the included study}

The literature retrieval process is shown in Fig. 1. A total of 162 studies were identified through key words retrieval. According to strict inclusion and exclusion criteria, 7 related studies were finally included for metaanalysis. The included literatures were evaluated with the NOS Literature Quality Evaluation Scale, and their scores all $\geqq 5$, suggesting that they belonged to literatures with better quality. The sample characteristics of the 7 case-control studies are shown in Table 1 and Table 2, including a total of 1415 cases and 1592 controls. Of these, 3 studies were related to hepatocellular carcinoma[6-8], 2 studies were related to liver cirrhosis[9, 10], and 2 studies involved primary liver cancer[11], and non-alcoholic fatty liver disease respectively[12]. Six studies focused on Asian population, one on African.

Table 1

Basic characteristics of the 7 case-control studies included.

\begin{tabular}{|c|c|c|c|c|c|c|c|c|}
\hline First author & Year & subject & Ethnicity & Disease type & $\begin{array}{l}\text { Genotyping } \\
\text { method }\end{array}$ & Case & Control & $\begin{array}{l}\text { Quality } \\
\text { score }\end{array}$ \\
\hline $\begin{array}{l}\text { Wu } \\
\text { Shisheng }\end{array}$ & 2012 & -- & Asian & $\begin{array}{l}\text { Primary liver } \\
\text { cancer }\end{array}$ & PCR-RFLP & 28 & 42 & 5 \\
\hline Wu Pengbo & 2015 & $\mathrm{HCC}$ & Asian & $\begin{array}{l}\text { non-alcoholic } \\
\text { fatty liver } \\
\text { disease }\end{array}$ & PCR-RFLP & 545 & 636 & 6 \\
\hline Liang & 2007 & $\mathrm{HCC}$ & Asian & liver cirrhosis & PCR-RFLP & 100 & 124 & 7 \\
\hline Okamoto & 2005 & $\mathrm{HCC}$ & Asian & liver cirrhosis & PCR-RFLP & 85 & 167 & 6 \\
\hline Yun Zhai & 2007 & $\mathrm{HCC}$ & Asian & $\begin{array}{l}\text { hepatocellular } \\
\text { carcinoma }\end{array}$ & $\begin{array}{l}\text { DNA } \\
\text { sequencing }\end{array}$ & 432 & 480 & 6 \\
\hline Samanoudy & 2014 & $\mathrm{HCC}$ & African & $\begin{array}{l}\text { hepatocellular } \\
\text { carcinoma }\end{array}$ & PCR-RFLP & 133 & 60 & 7 \\
\hline Okamoto & 2010 & $\mathrm{HCC}$ & Asian & $\begin{array}{l}\text { hepatocellular } \\
\text { carcinoma }\end{array}$ & PCR-RFLP & 92 & 83 & 6 \\
\hline
\end{tabular}


Table 2

Baseline data of the 7 case-control studies included.

\begin{tabular}{|lllllllll|}
\hline First author & \multirow{2}{*}{ Year } & \multirow{2}{*}{ Sample number } & \multicolumn{3}{l}{ Case } & \multicolumn{5}{c|}{ Control } \\
\cline { 5 - 9 } & & & CC & CT & TT & CC & CT & TT \\
\hline Wu Shisheng & 2012 & $28 / 42$ & 5 & 6 & 17 & 20 & 14 & 8 \\
\hline Wu Pengbo & 2015 & $545 / 636$ & 290 & 220 & 35 & 417 & 189 & 30 \\
\hline Liang & 2007 & $100 / 124$ & 70 & 24 & 6 & 86 & 30 & 8 \\
\hline Okamoto & 2005 & $85 / 167$ & 66 & 17 & 2 & 126 & 38 & 3 \\
\hline Yun Zhai & 2007 & $432 / 480$ & 338 & 94 & 0 & 369 & 111 & 0 \\
\hline Samanoudy & 2014 & $133 / 60$ & 34 & 38 & 61 & 21 & 23 & 16 \\
\hline Okamoto & 2010 & $92 / 83$ & 70 & 20 & 2 & 54 & 26 & 3 \\
\hline
\end{tabular}

2.2 Meta-analysis databases: Association between MMP-9-1562 C/T gene polymorphism and liver diseases

A total of 7 studies with 1415 cases and 1592 controls examined the relationship between MMP-9-1562 C/T gene polymorphism and the risk of liver diseases. The $\mathrm{I}^{2}$ of homozygous model and recessive model are $\leqq$ $50 \%$, so the fixed effect model was used for analysis. The $\mathrm{I}^{2}$ of heterozygote and dominant model are $>50 \%$, so the random effect model was used. After analysis of the pooled selected studies, there were significant association between $1562 \mathrm{C} / \mathrm{T}$ polymorphism of MMP-9 gene with liver diseases in homozygous model (Fig. 2. $\mathrm{TT}$ vs CC: $\mathrm{OR}=1.82,95 \% \mathrm{Cl}=1.28-2.59, P=0.0009)$ and recessive model (Fig. 3 . $\mathrm{TT}$ vs $\mathrm{CT}+\mathrm{CC}: \mathrm{OR}=$ $1.73,95 \% \mathrm{Cl}=1.24-2.41, P=0.01$ ), but not in heterozygote model (CT vs CC: $\mathrm{OR}=1.05,95 \% \mathrm{Cl}=0.76-1.45, P$ $=0.78$ ) and dominant model (TT $+\mathrm{CT}$ vs CC: $\mathrm{OR}=1.17,95 \% \mathrm{Cl}=0.82-1.67, P=0.38)$. The above results were shown in Table 3. (As TT genotype samples did not appear in the studies of Yun Zhai, we assume that the number of TT genotypes in this study is 1 .)

Table 3

Analysis results of MMP-9-1562 polymorphism and liver diseases risk.

\begin{tabular}{|lllll|}
\hline comparison & $\mathbf{I}^{2}$ & OR $95 \% \mathrm{Cl}$ & $\mathbf{z}$ & $\boldsymbol{p}$ \\
\hline TT vs CC & $37 \%$ & $1.82(1.28,2.59)$ & 3.32 & 0.0009 \\
\hline CT vs CC & $61 \%$ & $1.05(0.76,1.45)$ & 0.28 & 0.78 \\
\hline TT + CT vs CC & $72 \%$ & $1.17(0.82,1.67)$ & 0.88 & 0.38 \\
\hline TT vs CT + CC & $41 \%$ & $1.73(1.24,2.41)$ & 3.24 & 0.001 \\
\hline
\end{tabular}

\subsection{Analysis of publication bias}

In a meta-analysis, when there are less than 10 studies, the power of tests for funnel plot asymmetry is too low to distinguish chance from real asymmetry. Even so, we examined publication bias by a funnel plot with the effect size. The evaluation of publication bias of the four groups of models is shown in Fig. 4. The funnel 
plot data points of each model were not symmetric, hint that there may be publication bias, but also might cause from small sample size, methodological quality, and the presence of random error etc.

\subsection{Sensitivity analysis}

The results of homozygous models before literature exclusion showed that $\mathrm{I}^{2}=37 \%$, and that of recessive models before literature exclusion was $41 \%$. The source of heterogeneity was analyzed by sensitivity analysis. Results after each study was excluded in turn were shown in Table 4 and Table 5. It was found that $\mathrm{I}^{2}$ was reduced to $0 \%$ after Wu Shisheng's study was excluded from the two models, suggesting that the heterogeneity of the study results may be caused by this study, while the heterogeneity after the exclusion of the rest of the studies showed little change, indicating that these studies were relatively stable. Due to Wu Shisheng's small sample size, low literature quality and unknown sample source, it can be inferred that the heterogeneity between studies may be caused by sample size, quality of included literature and sample source.

Table 4

Sensitivity analysis of the relationship between homozygous model in MMP-9-1562 polymorphism and liver diseases

\begin{tabular}{|llll|}
\hline study on exclusion & $\mathbf{I}^{2}$ & OR ,95\% Cl after exclusion & P value after exclusion \\
\hline Samanoudy 2014 & $44 \%$ & $1.70[1.14,2.53]$ & 0.009 \\
\hline Liang 2007 & $37 \%$ & $1.97[1.36,2.86]$ & 0.0004 \\
\hline Okamoto 2005 & $47 \%$ & $1.84[1.29,2.64]$ & 0.0009 \\
\hline Okamoto 2010 & $35 \%$ & $1.92[1.34,2.75]$ & 0.0004 \\
\hline Wu Pengbo 2015 & $47 \%$ & $1.96[1.20,3.19]$ & 0.007 \\
\hline Yun Zhai 2007 & $47 \%$ & $1.83[1.29,2.62]$ & 0.0008 \\
\hline Wu Shisheng 2012 & $0 \%$ & $1.57[1.08,2.28]$ & 0.02 \\
\hline
\end{tabular}

Table 5

Sensitivity analysis of the relationship between recessive model in MMP-9-1562 polymorphism and liver diseases.

\begin{tabular}{|llll|}
\hline study on elimination & $\mathbf{I}^{2}$ & OR ,95\%Cl after elimination & $P$ value after elimination \\
\hline Samanoudy 2014 & $45 \%$ & $1.56[1.06,2.29]$ & 0.02 \\
\hline Liang 2007 & $43 \%$ & $1.85[1.30,2.62]$ & 0.0006 \\
\hline Okamoto 2005 & $50 \%$ & $1.75[1.25,2.45]$ & 0.001 \\
\hline Okamoto 2010 & $43 \%$ & $1.80[1.28,2.53]$ & 0.0007 \\
\hline Wu Pengbo 2015 & $43 \%$ & $2.06[1.32,3.22]$ & 0.001 \\
\hline Yun Zhai 2007 & $50 \%$ & $1.74[1.25,2.44]$ & 0.001 \\
\hline Wu Shisheng 2012 & $0 \%$ & $1.49(1.05,2.12)$ & 0.03 \\
\hline
\end{tabular}




\section{Discussion}

MMP-9 is a significant member of the family of MMPs, which belong to collagenase IV, widely expresses in the tissues and cells of our body, and mainly from monocytes, macrophages, neutrophils, fibroblasts, endothelial cells , and tumor cells[13]. MMP-9's gene locates on chromosome 20 q11.2-13.1, which is a vital component in degrading ECM and basement membrane [14]. Under normal physiological conditions, MMP-9 and TIMP work together to maintain the dynamic balance of the degradation and synthesis of ECM, and the stability of the tissue structure [15]. On the other hand, MMP-9 plays an important role in many physiological or pathological changes, such as inflammatory cells invasion and metastasis, vascular remodeling, organ fibrosis and cancer cell invasion and metastasis[16]. Under pathological conditions, the imbalance of ECM degradation and remodeling by MMP-9 affects the structure and function of various organs and cells, and changes the vascular microenvironment, thus promote the occurrence and development of various diseases $[17,18]$. Researches showed that MMP-9 is related to the susceptibility and development of some cardiovascular diseases such as coronary heart diseases ( $\mathrm{CHC}$ ), atherosclerosis, digestive system diseases such as gastric cancer, colorectal cancer, some liver diseases like NAFLD, HCC etc. [19-21]. Study[22] have found that MMP-9 has multiple gene polymorphism sites, among which the $-1562 \mathrm{C} / \mathrm{T}$ (rs3918242) SNP is located at the promoter polymorphism site, and is the most widely and deeply studied SNP site. In MMP-9$1562 \mathrm{~T}$ allele carriers, its level and activity of MMP-9 in plasma protein are higher than C allele carriers, and which is closely related to the susceptibility of various diseases, invasion and metastasis of tumor cells, etc. [23]. Therefore, MMP-9-1562 C/T polymorphism can be used as a potential biological detection index to evaluate the efficacy and prognosis of clinical individualized treatment and monitor the treatment process.

This study conducted a meta-analysis of 7 relevant literatures to explore the relationship between MMP-9$1562 \mathrm{C} / \mathrm{T}$ gene polymorphism and liver diseases. The results showed that the TT genotype may be a risk factor for primary hepatic cancer, hepatocellular carcinoma and nonalcoholic fatty liver disease. Research[24] showed that, compared with CC genotype carriers, the mRNA level, protein level and MMP-9 activity of -1562 T allele carriers were significantly increased, not only can promote the proliferation, activation, and migration of hepatic stellate cells (HSC), but also stimulate the development of liver inflammation and fibrosis[25], leading to vascular remodeling of organs and changes of microenvironment. So we can infer that, compared with non-carriers, the MMP- 9 transcription and translation level in MMP-9-1562 T allele carriers was increased significantly, which promote the activation of HSC, and make HSC excessive secrete inflammatory factors and fibrosis factors such as IL-1, transforming growth factor (TGF- $\beta$ ), and platelet-derived growth factor (PDGF), which accelerate the imbalance of ECM degradation and synthesis, leading to excessive deposition of ECM in the liver, finally resulting in NAFLD from simple fatty liver to fatty liver fibrosis pathological change. At the same time, the overexpression of MMP-9 activates TGF- $\beta$ /SMAD, PI3K/AKT and other signal transduction pathways, which stimulate the proliferation of HSC and inhibit its apoptosis, accelerating the invasion and metastasis of inflammatory cells or cancer cells, enhancing vascular remodeling and changes, and thus lead to the occurrence and development of primary liver cancer, hepatocellular carcinoma and other liver cancers.

There are still some limitations in this study: (1) the number of references included in this study is small, and the number of research samples is small, which limits the demonstration intensity of the results of the 
systematic evaluation; (2) The liver diseases included in this study refer to primary liver cancer, non-alcoholic fatty liver, liver cirrhosis, and hepatocellular carcinoma; However, there was only one reference for each of the first two diseases, so there may be some bias and cannot represent the general situation.

With the development of society, liver disease has become a clinically urgent problem to be solved. This study suggests that in MMP-9-1562 C/T gene polymorphism, the TT genotype may be one of the risk factors for PHC, HCC and NAFLD. This means that patients with NAFLD with MMP-9-1562 TT genotype may have a higher risk of developing liver cancer, so early intervention should be carried out to prevent the occurrence of liver cancer in clinical practice; active diagnosis and treatment should be carried out for patients with HCC or PLC with TT genotype, and corresponding gene therapy should be explored to improve the quality of life and prognosis of cancer patients. Therefore, in the future clinical treatment of chronic liver diseases, more indepth researches are needed to explore the important role and mechanism of MMP-9-1562 gene polymorphism in the occurrence and development of liver diseases, so as to provide basis for individualized disease monitoring, curative effect evaluation and prognosis of liver diseases, and also provide help and new ideas for the study of liver diseases mechanism.

\section{Conclusions}

Our meta-analysis suggested that the TT genotype of MMP-9-1562 C/T polymorphism might be associated with the risk of nonalcoholic fatty liver disease, hepatocellular carcinoma, and primary liver cancer. If these diseases are present, screening genotype of MMP-9 and aggressive treatment of the primary disease is necessary.

\section{List Of Abbreviations}

ECM, extracellular matrix; MMP-9, Matrix Metalloproteinase-9; SNP, single nucleotide polymorphism; OR, odds ratio; $\mathrm{Cl}$, confidence interval; $\mathrm{HCC}$, hepatocellular carcinoma; NAFLD, non-alcoholic fatty liver disease; PLC, primary liver cancer

\section{Declarations}

\section{Ethics approval and consent to participate}

Not applicable

\section{Consent for publication}

Not applicable

\section{Availability of data and material}

All data generated or analyzed in this study are included in this published article.

\section{Conflicts of interest/Competing interests}


The authors declare that there are no conflict of interests.

\section{Funding}

Research grants from Demonstration of comprehensive prevention and treatment of liver disease in high incidence area of liver disease in Liaoning Province, China(2019JH2/10300031-02)

Authors' contributions

Yang Tianling wrote the paper and made statistical analysis; Chen Shitong and Li Shumeng helped with statistical processing; Ji Yangtao guided the paper writing and revised the paper.

Acknowledgments

The authors would like to thank all the participants and the support of funding.

\section{References}

1. Torbenson M, Washington K: Pathology of liver disease: advances in the last $\mathbf{5 0}$ years. Human pathology 2020, 95:78-98.

2. Chinese Society of Hepatology CMA, Chinese Society of Gastroenterology CMA, Chinese Society of Infectious Diseases CMA: Consensus on the diagnosis and treatment of hepatic fibrosis (2019). Journal of digestive diseases 2020, 21(3):127-138.

3. Tan QR, Li H, Yang Q, Han PL: Research progress on the role of matrix metalloproteinases and their specific inhibitors in the formation of liver fibrosis. Shandong Medical Journal 2017, 57(34):109-111.

4. He T, Wang J, Wang XL, Deng WS, Sun P: Association between the Matrix Metalloproteinase-9 rs3918242 Polymorphism and Ischemic Stroke Susceptibility: A Meta-Analysis. Journal of stroke and cerebrovascular diseases : the official journal of National Stroke Association 2017, 26(5):1136-1143.

5. Hu C, Weng F, Li L, Dai W, Yan J, Peng L, Zhou R: Association between MMP-9 -1562 C/T polymorphism and susceptibility to digestive cancers: A meta-analysis. Gene 2018, 673:88-94.

6. El Samanoudy A, Monir R, Badawy A, Ibrahim L, Farag K, El Baz S, Alenizi D, Alenezy A: Matrix metalloproteinase-9 gene polymorphism in hepatocellular carcinoma patients with hepatitis $\mathrm{B}$ and $\mathrm{C}$ viruses. Genetics and molecular research : GMR 2014, 13(3):8025-8034.

7. Okamoto K, Ishida C, Ikebuchi Y, Mandai M, Mimura K, Murawaki Y, Yuasa I: The genotypes of IL-1 beta and MMP-3 are associated with the prognosis of HCV-related hepatocellular carcinoma. Internal medicine (Tokyo, Japan) 2010, 49(10):887-895.

8. Zhai Y, Qiu W, Dong XJ, Zhang XM, Xie WM, Zhang HX, Yuan XY, Zhou GQ, He FC: Functional polymorphisms in the promoters of MMP-1, MMP-2, MMP-3, MMP-9, MMP-12 and MMP-13 are not associated with hepatocellular carcinoma risk. Gut 2007, 56(3):445-447.

9. Okamoto K, Mimura K, Murawaki Y, Yuasa I: Association of functional gene polymorphisms of matrix metalloproteinase (MMP)-1, MMP-3 and MMP-9 with the progression of chronic liver disease. Journal of gastroenterology and hepatology 2005, 20(7):1102-1108. 
10. Liang Q, Liu Q, Li CC, Xue FX, Li Z, Yan HM, Fu W: Polymorphisms of matrix metalloproteinase-9 in hepatitis B-related cirrhosis. J Clin Hepatol 2007, 10(04):229-231.

11. Wu SS, Shao LH, Li SR, Zhang F, Xie H, Zhang CX, Liu GP: Correlation of MMP-9 and MMP-2 Gene SNPs with Hepatocellular Carcinoma Invasion and Metastasis. Cancer prevention and treatment research 2012, 39(06):683-686.

12. Wu P, Shu Y, Guo F, Luo H, Zhang G, Tan S: Association between patatin-like phospholipase domaincontaining protein 3 gene rs738409 polymorphism and non-alcoholic fatty liver disease susceptibility: a meta-analysis. Zhonghua liu xing bing xue za zhi = Zhonghua liuxingbingxue zazhi 2015, 36(1):78-82.

13. Ling Z, Rongyong M: Research progress of butylphthalide in the treatment of acute cerebral infarction and its effect on serum MMP-9. 2018, 34(10):1525-1528.

14. Szychowski KA, Wójtowicz AK, Gmiński J: Impact of Elastin-Derived Peptide VGVAPG on Matrix Metalloprotease-2 and -9 and the Tissue Inhibitor of Metalloproteinase-1, $-2,-3$ and -4 mRNA Expression in Mouse Cortical Glial Cells In Vitro. Neurotoxicity research 2019, 35(1):100-110.

15. Ugarte-Berzal E, Vandooren J, Bailón E, Opdenakker G, García-Pardo A: Inhibition of MMP-9-dependent Degradation of Gelatin, but Not Other MMP-9 Substrates, by the MMP-9 Hemopexin Domain Blades 1 and 4. The Journal of biological chemistry 2016, 291(22):11751-11760.

16. Wu CW, Chen P, Long SS, Liu HL: Association of matrix metalloproteinases $₫ 9$ gene polymorphisms at

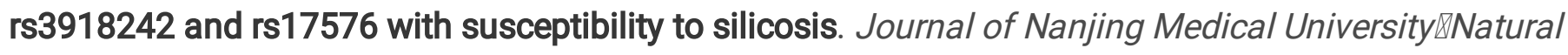

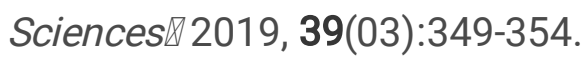

17. Łukaszewicz-Zając M, Szmitkowski M, Litman-Zawadzka A, Mroczko B: Matrix Metalloproteinases and Their Tissue Inhibitors in Comparison to Other Inflammatory Proteins in Gastric Cancer (GC). Cancer investigation 2016, 34(7):305-312.

18. Verma S, Kesh K, Gupta A, Swarnakar S: An Overview of Matrix Metalloproteinase 9 Polymorphism and Gastric Cancer Risk. Asian Pacific journal of cancer prevention : APJCP 2015, 16(17):7393-7400.

19. Cui N, Hu M, Khalil RA: Biochemical and Biological Attributes of Matrix Metalloproteinases. Progress in molecular biology and translational science 2017, 147:1-73.

20. Gao N, Guo T, Luo H, Tu G, Niu F, Yan M, Xia Y: Association of the MMP-9 polymorphism and ischemic stroke risk in southern Chinese Han population. BMC Neurol 2019, 19(1):67.

21. Krishnaveni D, Bhayal AC, Sri Manjari K, Vidyasagar A, Uma Devi M, Ramanna M, Jyothy A, Nallari P, Venkateshwari A: MMP 9 Gene Promoter Polymorphism in Gastric Cancer. Indian journal of clinical biochemistry : IJCB 2012, 27(3):259-264.

22. Huang H: Matrix Metalloproteinase-9 (MMP-9) as a Cancer Biomarker and MMP-9 Biosensors: Recent Advances. Sensors (Basel, Switzerland) 2018, 18(10).

23. Yang W, Lu J, Yang L, Zhang J: Association of Matrix Metalloproteinase-9 Gene -1562C/T Polymorphism with Essential Hypertension: A Systematic Review and Meta-Analysis Article. Iranian journal of public health 2015, 44(11):1445-1452.

24. Latronico T, Mascia C, Pati I, Zuccala P, Mengoni F, Marocco R, Tieghi T, Belvisi V, Lichtner M, Vullo V et al: Liver Fibrosis in HCV Monoinfected and HIV/HCV Coinfected Patients: Dysregulation of Matrix 
Metalloproteinases (MMPs) and Their Tissue Inhibitors TIMPs and Effect of HCV Protease Inhibitors. International journal of molecular sciences 2016, 17(4):455.

25. Ye S: Polymorphism in matrix metalloproteinase gene promoters: implication in regulation of gene expression and susceptibility of various diseases. Matrix biology : journal of the International Society for Matrix Biology 2000, 19(7):623-629.

\section{Figures}
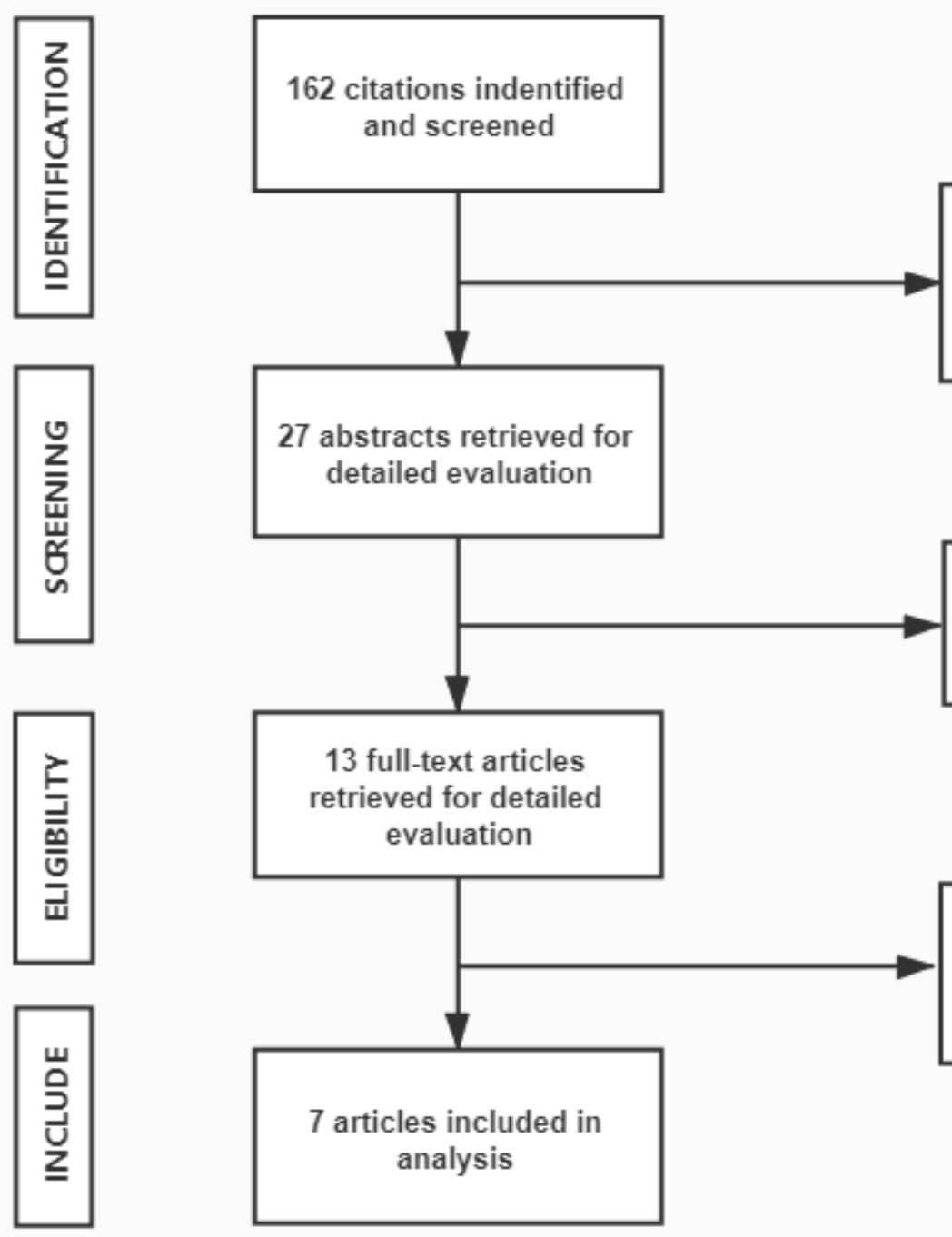

135 excluded

15 were obviously irrelevant 118 not liver disease

2 review
14 excluded

3 not for MMP-9 research

11 were meta-analysis
6 excluded

4 not-1562 polymorphism

2 control group did not meet the requirements

Figure 1

Flow chart of the identified studies. 


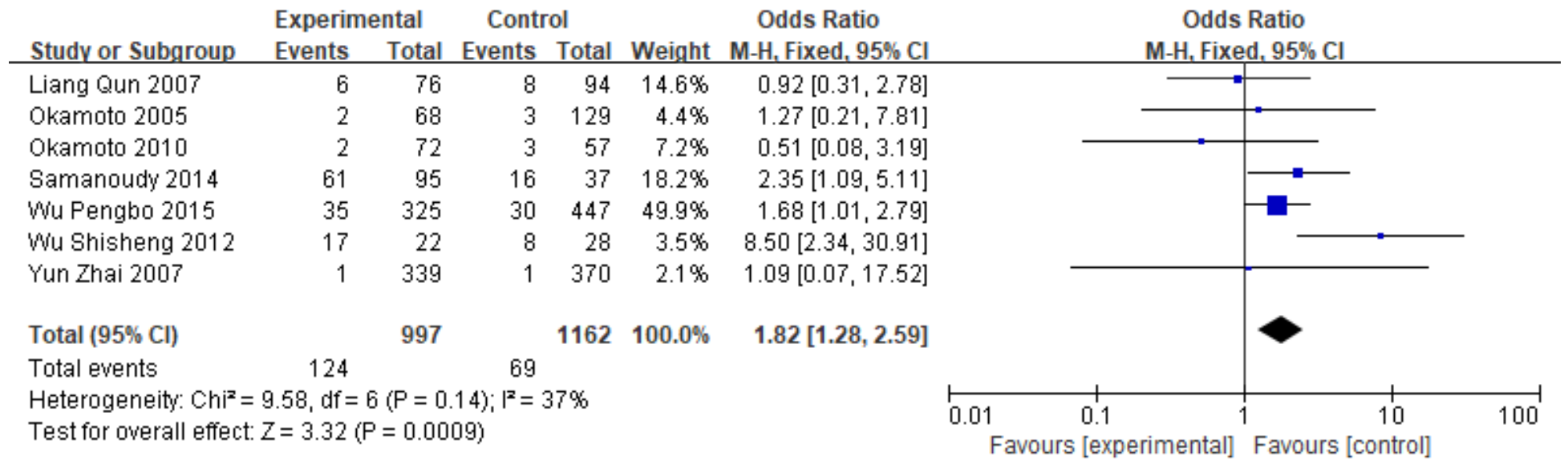

\section{Figure 2}

Forest plot of MMP-9 -1562 C/T polymorphism in liver diseases risk under homozygous model.

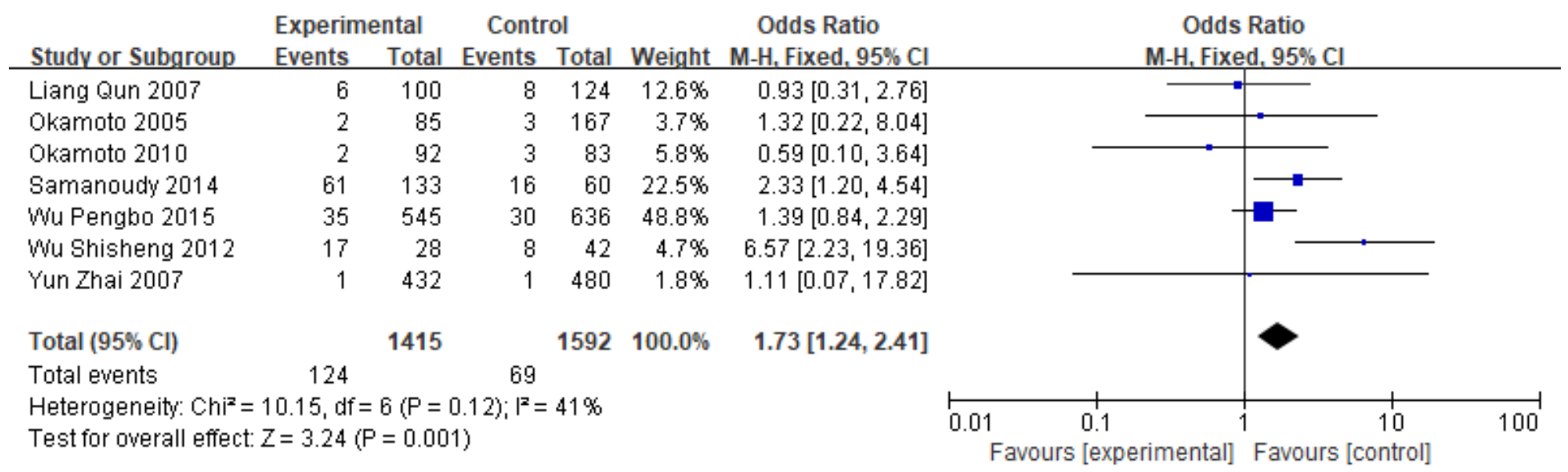

\section{Figure 3}

Forest plot of MMP-9 -1562 C/T polymorphism in liver diseases risk under recessive model. 
(A)

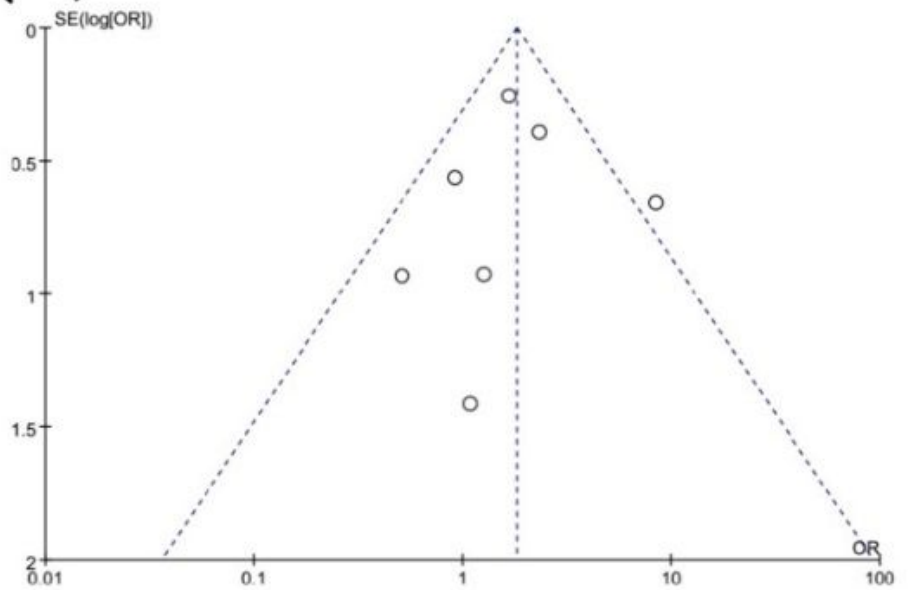

(C)

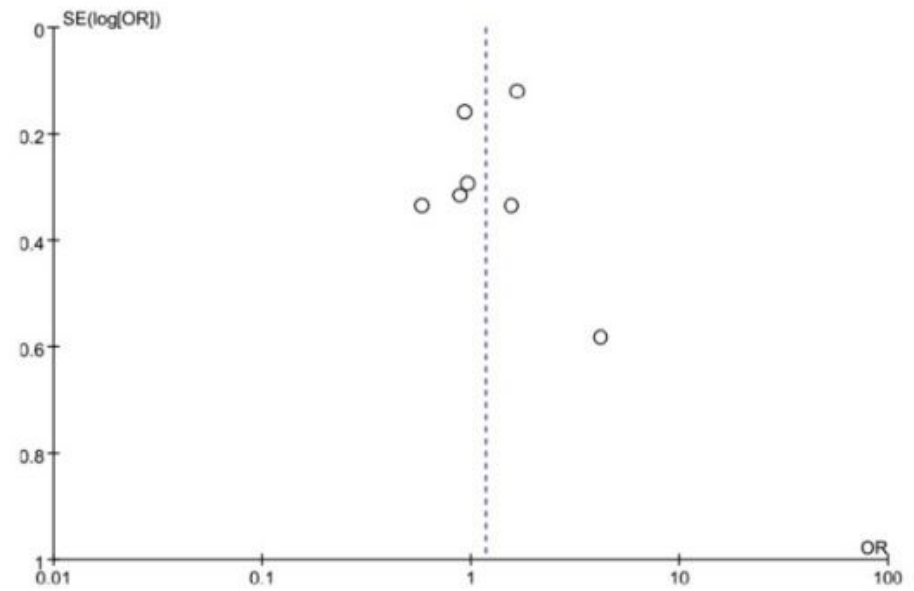

(B)

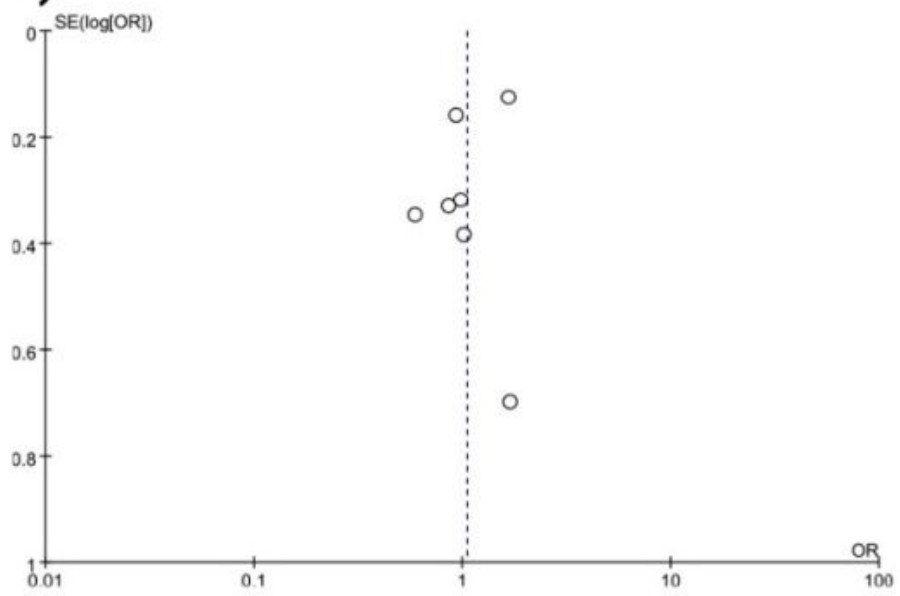

(D)

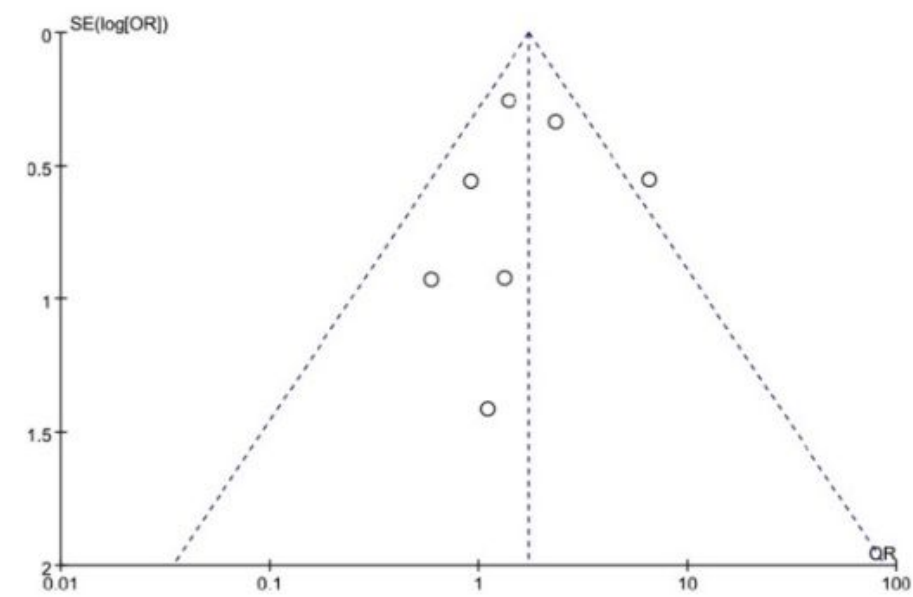

Figure 4

Funnel plot data points (A). Funnel plot of TT vs CC homozygous model; (B). Funnel plot of CT vs CC heterozygous model; (C). Funnel plot of TT+CT vs CC dominant model; (D). Funnel plot of TT vs CT+CC recessive model. OR: odds ratio; $S E[\log (\mathrm{OR})]$ : a standard error of the logarithm of odds ratio value. 